\title{
P01-9-18 Poster session
}

\section{Study of antibacterial effect of lupinifolin on cytoplasmic membrane of Staphylococcus aureus by flow cytometry}

\author{
Kamol Yusook
}

Public Health, Phetchabun Rachabhat University, Thailand

Nowadays, flow cytometry (FCM), was widely applied to determine the physiological changes in bacterial cells. FCM is an automated technique with time-saving, accurate and sensitive. Furthermore, as thousands of bacterial cells can be processed in a second, statistics are improved. Therefore, this technique can provide invaluable information regarding the alteration of bacterial cells when treating with antibacterial agents either antibiotics or phytochemicals. We applied the FCM to investigate the mechanism of action of lupinifolin against Staphylococcus aureus (S. aureus). According to the previous study, lupinifolin from Derris reticulata Craib. was extracted with hexane by Soxhlet extractor and purified by crystallization. Then, the yellow needle-shaped lupinifolin crystals were identified and confirmed by nuclear magnetic resonance (NMR) spectra mass spectrometry and Liquid chromatography mass spectrometry (LC/MS), respectively. In addition, we determined antibacterial activity of lupinifolin including investigation of its mechanism of action. The lupinifolin showed minimum inhibitory concentration (MIC) of $8 \mu \mathrm{g} / \mathrm{ml}$ against $\mathrm{S}$. aureus. The FCM was performed to determine the alteration of cytoplasmic membrane (CM) permeability of S. aureus. By using Propidium iodide (PI) 5 $\mu \mathrm{g} / \mathrm{ml}$ as an indicator for bacterial membrane integrity, it was found that the bacterial CM permeability was effected by lupinifolin with the MIC of $8 \mu \mathrm{g} / \mathrm{ml}$ comparable to the control. This indicated the significantly decreased integrity of bacterial membrane. Additionally, DNA laddering assay was carried out to evaluate apoptosis in bacterial cells. It was revealed that the lupinifolin has no effect on DNA fragmentation.

Currently, S. aureus is the most serious problem threatening to medical implications. Therefore, the research and development of new therapies to conquer this opportunistic bacterium are urgently needed. To reveal the insights regarding the mechanism of action of lupinifolin against S. aureus, the FCM can be employed. In accordance with the advantages of FCM mentioned above, it becomes a powerful technique which can be applied to determine the physiological changes in bacterial cells in response to antibacterial agents. 